\title{
Association Between Passive Hip Range of Motion and Pitching Kinematics in High School Baseball Pitchers
}

\author{
Hillary A. Plummer, PhD, ATC ${ }^{1}$ (D) ${ }^{\text {, }}$ Nicole M. Bordelon, MS, CSCS², Kyle W. Wasserberger, MS, CSCS² (D, Tyler J. Opitz, \\ PT, DPT, CSCS ${ }^{3}$, Adam W. Anz, MD ${ }^{4}$, Gretchen D. Oliver, PhD, ATC ${ }^{2}$ \\ ${ }^{1}$ Warfighter Performance Group, U.S. Army Aeromedical Research Laboratory, ${ }^{2}$ School of Kinesiology, Auburn University, ${ }^{3}$ Physical Therapy, \\ Andrews Institute for Orthopedics \& Sports Medicine, ${ }^{4}$ Andrews Institute for Orthopedics \& Sports Medicine \\ Keywords: biomechanics, injury, pitching \\ https://doi.org/10.26603/001c.27625
}

\section{International Journal of Sports Physical Therapy}

Vol. 16, Issue 5, 2021

\section{Background}

Limitations in passive hip range of motion (PROM) may negatively affect pitching mechanics in baseball pitchers. Understanding the relationships between PROM and mechanics can assist in the development of injury prevention protocols.

\section{Purpose}

The purpose of this study was to examine the association of hip rotational PROM with pelvis and trunk rotation during pitching in high school baseball pitchers.

Study Design: Cross-sectional.

\section{Methods}

Twenty-five healthy high school baseball pitchers volunteered (15.9 \pm 1.1 years; $180.4 \pm$ $5.5 \mathrm{~cm} ; 75.4 \pm 9.3 \mathrm{~kg}$ ). Seated passive hip internal rotation (IR) and external rotation (ER) PROM were measured using a digital inclinometer. Total PROM was calculated (IR+ER). Pitching biomechanical data were collected with a 3-dimensional electromagnetic tracking system while pitchers threw fastballs. Simple linear regressions were performed to examine the association between hip IR, ER, and total PROM with pitching kinematics at foot contact including stride length, pelvis rotation, and trunk rotation.

\section{Results}

Only one significant association in PROM and kinematics was observed. Drive leg hip IR PROM was associated with trunk rotation angle $[\mathrm{F}(1,24)=4.936, \mathrm{p}=0.036]$, with an $\mathrm{R}^{2}=$ 0.177. Drive leg total PROM was not associated trunk rotation angle $[F(1,24)=4.144, p=$ $0.053]$ with an $\mathrm{R}^{2}=0.153$.

\section{Conclusions}

Increased drive leg hip IR PROM was associated with decreased trunk rotation towards home plate. Hip total PROM and ER were not related to pitching mechanics.

\section{Level of Evidence}

2

\section{INTRODUCTION}

Baseball pitching is a dynamic motion requiring optimal proximal to distal sequencing of the kinetic chain to produce maximal ball velocity. ${ }^{1,2}$ Proper pitching mechanics, joint passive range of motion (PROM), core stability, and

\footnotetext{
a Corresponding author:

U.S. Army Aeromedical Research Laboratory

Ft. Rucker, AL 36362

Oak Ridge Institute for Science \& Education

Oak Ridge, TN

hplummer47@gmail.com
} 
strength are critical in the transfer of energy from the lower extremities to the trunk, shoulder, elbow, wrist, and finally hand before ball release. ${ }^{1-3}$ At foot contact of the pitch, the stride foot should be planted slightly towards third base (for a right handed pitcher) and pointed slightly inward. ${ }^{4}$ Hip PROM may contribute to lower extremity positioning during pitching therefore it is important to examine the association between these variables. Improper stride foot positioning can cause the pelvis and trunk to rotate towards the catcher too early in the pitching motion, which leads to improper segmental sequencing. ${ }^{4}$ Improper segmental sequencing of the lower extremities can lead to compensations at the trunk and upper extremity that result in increased upper extremity forces. ${ }^{5}$ Lower extremity mechanical or functional deficits can result in compensation patterns in the trunk and upper extremity to maintain ball velocity. ${ }^{4,6}$ During pitching segmental power of trunk motion contributes to the development of ball velocity and elbow valgus load and ball velocity. ${ }^{5}$ High school pitchers have been reported to have different patterns of segmental sequencing compared to professional pitchers despite having similar elbow valgus load relative to size. ${ }^{5}$ Over time high shoulder and elbow loads may contribute to tissue breakdown and injury. ${ }^{7-9}$

Hip PROM is one functional measure that can influence pitching mechanics. The repetitive nature of the pitching motion leads to adaptations in the hip joint tissues, often leading to changes in hip PROM. ${ }^{10-13}$ Rob et al. determined professional pitchers display less hip external rotation (ER), internal rotation (IR), and total PROM in the stride leg compared to the drive leg. ${ }^{10}$ The results also showed decreased stride leg total PROM was associated with lower trunk separation velocity while increased total drive leg PROM has been associated with a more open pelvis at foot contact. ${ }^{10}$ It has also been speculated in youth that decreased stride leg hip IR PROM contributes to insufficient trunk rotation during pitching and may lead to increased forces about the shoulder and elbow, contributing to tissue breakdown and pain. 14,15

A comprehensive understanding of the relationships between hip PROM and pitching mechanics is also needed in high school pitchers. ${ }^{10}$ The findings of this study can contribute to developing rehabilitation programs that target hip PROM deficits to improve pitching performance and decrease the risk of injury. The purpose of this study was to examine the association of hip rotational PROM with pelvis and trunk rotation during pitching in high school baseball pitchers. It was hypothesized pitchers with lower hip rotational PROM would have increased trunk and pelvis rotation towards home plate at foot contact.

\section{METHODS}

Twenty-five healthy high school baseball pitchers volunteered $(15.9 \pm 1.1$ years; $180.4 \pm 5.5 \mathrm{~cm} ; 75.4 \pm 9.3 \mathrm{~kg} ; 8.3 \pm$ 3.2 years of competitive baseball experience; $n=17$ righthand dominant). Two pitchers were tested in the spring (January-April), two were tested in the summer (May-August), and the rest were tested in the fall (September-December). Inclusion criteria consisted of no injury in the prior six months and no history of surgery to the lower or

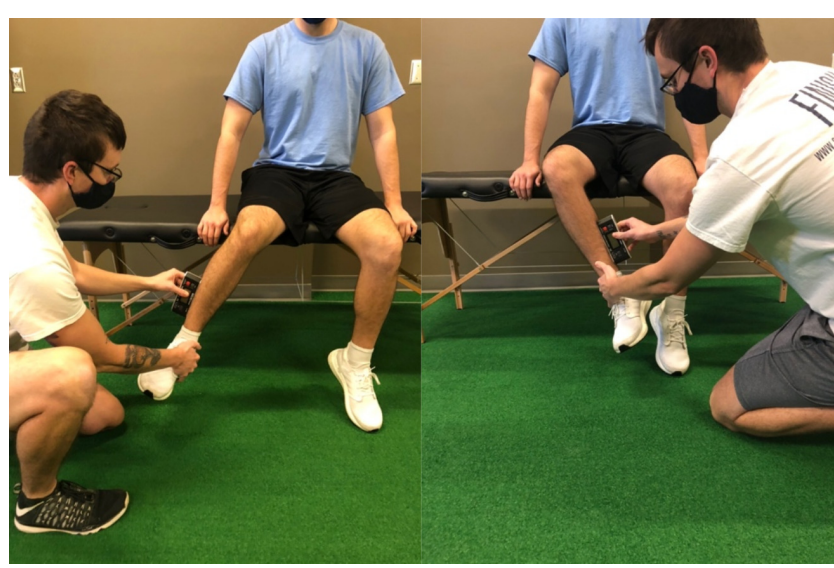

Figure 1. Measurements of hip passive internal and external rotation range of motion.

upper extremities. At the start of each data collection, testing procedures were explained and informed consent and parental assent were obtained. The study was approved by the Auburn University Institutional Review Board.

An a priori power analysis was conducted using $G^{*}$ Power3 to test the difference between two dependent means using a two-tailed test, an alpha of 0.05 , and a power of 0.8 . An effect size of 2.4 was calculated from previously reported total hip PROM data on the mean difference between the stride and drive leg $\left(27.8^{\circ}\right)$ and standard deviation $\left(10.5^{\circ}\right)$ in professional pitchers. ${ }^{10}$ It was estimated that a sample size of four participants would be needed.

After reviewing testing procedures, participant hip rotational PROM (IR and ER) was assessed. Hip rotational PROM was measured passively with the participant in a seated position. Their knees were flexed to $90^{\circ}$ allowing the legs to comfortably hang off the edge of the table with their hands resting comfortably on the table to assist with trunk stabilization. ${ }^{10,16-18}$ The hip was positioned in $90^{\circ}$ of flexion by placing a towel under the femur, and a digital inclinometer was aligned along the soft tissue contour of the participant's tibia (Figure 1). The examiner supported the femur to eliminate accessory motion and passively rotated the hip until capsular end-feel was achieved. At the point of a firm capsular end-feel without the production of accessory hip movement (hip hiking), the PROM measurement was recorded. ${ }^{10,11,17}$ Total PROM was calculated (IR+ER). The same examiner measured each participants PROM. Testretest reliability for the examiner was established prior to the study. Excellent intra-rater reliability for the hip was observed ICC $_{(3, \mathrm{k})}$ of 0.92 to 0.95 for all measurements). Minimal detectable change (MDC) values were calculated for hip IR and ER PROM, $\mathrm{MDC}_{95}$ was $5.6^{\circ}$ and $4.7^{\circ}$, respectively.

Fourteen electromagnetic sensors (trakSTAR, Ascension Technologies Inc.; Burlington, VT, USA) were placed on the skin using double-sided cohesive tape and were then wrapped in flexible adhesive tape to secure sensor position at the following sites: (1) posterior aspect of the first thoracic vertebrae, (2) posterior aspect of the pelvis at the first sacral vertebrae, (3-4) flat, broad portion of the acromion on the bilateral scapula, (5-6) lateral aspect of bilateral up- 
per arm at the deltoid tuberosity, (7-8) posterior aspect of the bilateral distal forearm, (9-10) lateral aspect of bilateral upper leg centered between the greater trochanter and the lateral condyle of the knee, (11-12) lateral aspect of the bilateral lower leg centered between the head of the fibula and lateral malleolus, (13) dorsal aspect of the second metatarsal of the stride foot, and (14) dorsal aspect of the third metacarpal of the pitching hand. A fifteenth moveable sensor was attached to a plastic stylus and used for digitization of bony landmarks. ${ }^{19,20}$ The stylus was used to digitize the anterior superior iliac spine and posterior superior iliac spine of the pelvis and used Bell offsets to calculate hip joint centers. ${ }^{21}$ The rotation method was used to calculate shoulder joint centers. ${ }^{22}$ Ankle and knee joints were defined as the midpoint between the medial and lateral malleoli and femoral condyles respectively. The digitized space between C-7 and T12-L1 defined the spinal column and a validated rotation method, where the hip or shoulder is stabilized and rotated to 10 different positions in a circular motion, was used to estimate hip and shoulder joint centers. ${ }^{23}$

Sensor position and orientations were collected at 240 $\mathrm{Hz}$ using the trakSTAR, which was synchronized with The MotionMonitor software (Innovative Sports Training; Chicago, IL, USA). Sensor positions and orientations were transformed to locally-based coordinate systems for the captured body segments based on the recommended definitions of reporting human joint motions standardized by the International Society of Biomechanics. ${ }^{20}$ The world axis was defined with the positive $\mathrm{Y}$ axis pointing vertically, the positive $\mathrm{X}$ axis pointing in the direction of movement, and the positive $\mathrm{Z}$ axis orthogonal to $\mathrm{X}$ and $\mathrm{Y}$ and pointing to the right. Orientation for the pelvis and trunk were referenced to the world axis using a ZX'Y" Euler rotation sequence. A $4^{\text {th }}$-order Butterworth filter with a cutoff frequency of $13.4 \mathrm{~Hz}$ was applied independently to all raw data along each global axis. ${ }^{24}$ Data were analyzed at foot contact of the pitching motion (Figure 2).

Pitchers were allotted unlimited time to perform their pre-competition pitching warm-up. Warm-up routine and time was subjective to each pitcher and depended on their own individual routines to replicate game day performance. ${ }^{25}$ Pitchers instructed to warm-up so that they would be prepared to make maximal effort pitches once testing began. All pitchers included maximal effort pitches from a mound, at regulation distance, in their warm-up. After warming up, pitchers threw three maximal-effort four-seam fastballs, from a mound, to a catcher at regulation distance $(18.5 \mathrm{~m})$. Only pitches that were deemed to be strikes, by an investigator with prior baseball or softball experience, were used for analysis. The average data at foot contact, for the three trials, was calculated for each variable. The drive leg was defined as the ipsilateral hip to the throwing arm and the stride leg was contralateral to the throwing arm. Ball velocity was recorded by a calibrated radar gun (Stalker Pro II; Stalker Radar; Plano TX, USA).

\section{STATISTICAL ANALYSIS}

A paired sample t-test was used to assess differences in PROM between drive and stride legs. Simple linear regressions were performed to examine the association between

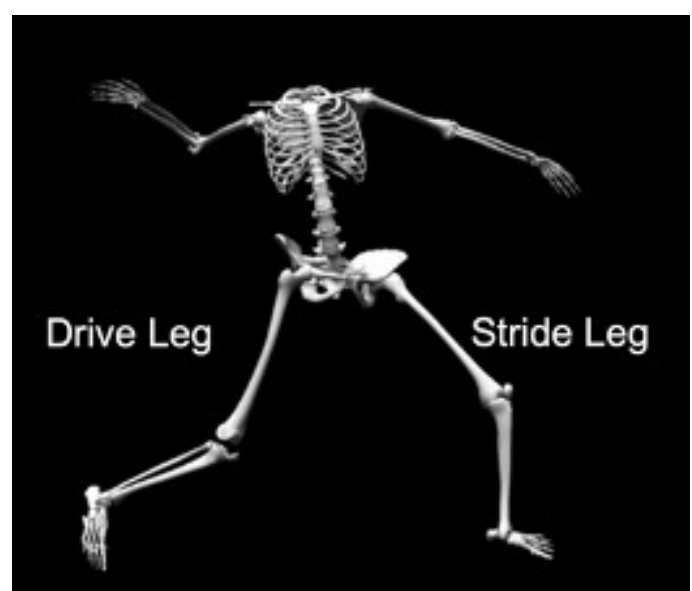

Figure 2. Foot contact of the pitching motion for a right-handed pitcher.

hip IR, ER, and total PROM with pitching kinematics at foot contact including stride length, pelvis rotation, and trunk rotation. Statistical significance was set $a$ priori at $p<0.05$ and all analyses were performed using IBM SPSS Statistics Version 25.0 software (International Business Machines Corp., Armonk, NY, USA).

\section{RESULTS}

Mean ball velocity was $31.3 \pm 2.1 \mathrm{~m} / \mathrm{s}(70.1 \pm 4.6 \mathrm{mph})$. Means and standard deviations can be found for kinematic data at foot contact and hip range of motion values in Table 1. Only one significant association in PROM and kinematics was observed. Drive leg hip IR PROM was associated with trunk rotation angle $[\mathrm{F}(1,24)=4.936, \mathrm{p}=0.036]$, with an $\mathrm{R}^{2}$ $=0.177$ (Figure 3 ). Drive leg total PROM was not associated trunk rotation angle $[\mathrm{F}(1,24)=4.144, \mathrm{p}=0.053]$ with an $\mathrm{R}^{2}=$ 0.153 . Lastly, no significant differences were found between drive and stride legs for IR, ER, and total hip PROM values.

\section{DISCUSSION}

The purpose of the study was to examine the association of hip rotational PROM with lower extremity and trunk kinematics at foot contact in high school baseball pitchers. The findings partially support the hypothesis that lower hip PROM would be associated with altered trunk rotation mechanics. Drive leg hip IR PROM in high school baseball pitchers predicted trunk rotation positioning at foot contact, where drive leg hip IR explained $17.7 \%$ of the variance in trunk rotation. Pitchers with increased drive leg hip IR PROM had decreased trunk rotation towards home plate. No other significant differences were observed between hip PROM and pitching kinematic parameters.

The drive leg functions to propel the body towards home plate and adequate hip internal rotation is needed to position the stride leg foot. ${ }^{11,26}$ Weight is transferred from the drive leg to the stride leg at stride foot contact and the stride leg acts to provide stability as the pitch progresses to a position of single-leg support. Adequate hip internal rota- 
Table 1. Mean (SD) for kinematic data at foot contact and passive range of motion.

\begin{tabular}{|c|c|c|c|c|c|}
\hline \multicolumn{6}{|c|}{ Range of Motion } \\
\hline \multicolumn{3}{|c|}{ Stride Leg } & \multicolumn{3}{|c|}{ Drive Leg } \\
\hline Total PROM & IR PROM & ER PROM & Total PROM & IR PROM & ER PROM \\
\hline $57.0(11.0)$ & $24.2(6.8)$ & $32.9(6.9)$ & $58.2(13.4)$ & $25.1(8.2)$ & $33.1(6.6)$ \\
\hline \multicolumn{6}{|c|}{ Kinematics } \\
\hline Pelvis Rotation $\left({ }^{\circ}\right)$ & & \multicolumn{2}{|c|}{ Trunk Rotation $\left({ }^{\circ}\right)$} & \multicolumn{2}{|c|}{ Stride Length $(\mathrm{m})$} \\
\hline$-61.1(11.9)$ & & \multicolumn{2}{|l|}{$-98.4(17.7)$} & \multicolumn{2}{|l|}{$1.2(0.09)$} \\
\hline
\end{tabular}

Trunk \& pelvis rotation: smaller values = greater rotation towards home plate $\left(0^{\circ}\right.$ of rotation is facing home plate, $180^{\circ}$ is facing centerfield $)$

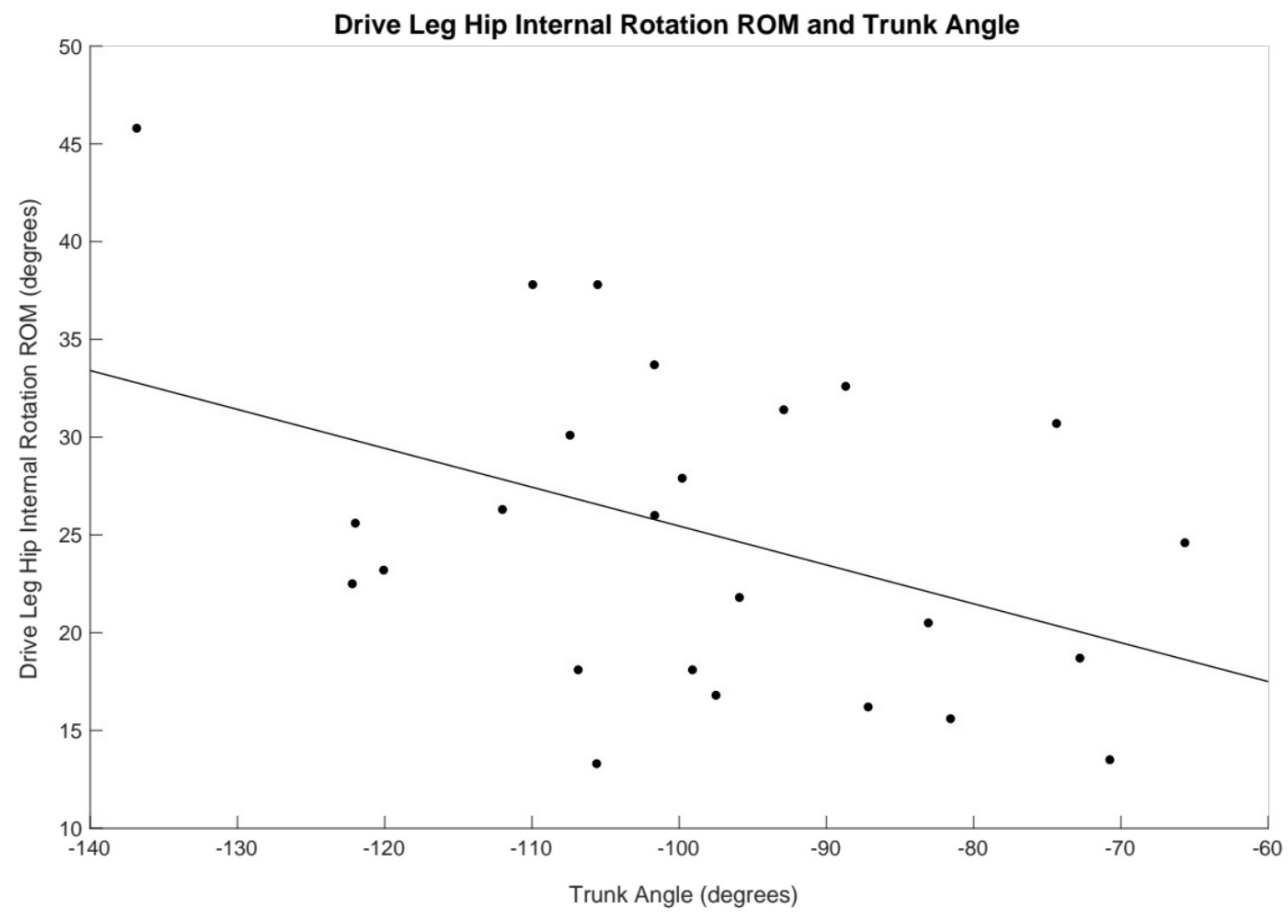

Figure 3. The relationship between drive leg hip internal rotation PROM and trunk rotation angle. $0^{\circ}$ indicates the shoulders open and facing the catcher.

tion of the stride leg is required to allow the trunk to properly rotate after pelvis rotation optimizing proximodistal energy transfer. ${ }^{11,26}$ Previous literature has speculated that decreased stride leg hip IR PROM contributes to insufficient trunk rotation during pitching and may lead to increased forces about the shoulder and elbow, contributing to tissue breakdown and pain. ${ }^{14,15}$ However, the current study determined increased drive leg hip IR PROM was associated with decreased trunk rotation towards home plate at foot contact. Less trunk rotation at foot contact may beneficial since prior research suggests too much trunk rotation towards home plate at foot contact may result in the throwing arm lagging behind the body which is known to increase forces at the shoulder and elbow. Therefore, sufficient drive leg hip IR PROM may support proper trunk rotation mechanics at foot contact and potentially reduce injurious upper extremity forces.

Reducing injurious upper extremity forces requires an understanding of the kinematics and other potential factors that contribute to the increased forces. Early trunk rotation, increased shoulder ER, and decreased elbow flexion during pitching increase elbow valgus loads. ${ }^{9}$ The relationship between hip PROM and kinematics that contribute to increased elbow valgus loads has been examined in collegiate pitchers. ${ }^{27}$ Stride leg hip total PROM is associated with maximum shoulder ER and drive leg ER and total PROM is associated with trunk angular velocity during pitching. ${ }^{27}$ It is important to note that only seven collegiate pitchers were examined and the findings may have been due to chance because the study was underpowered. More research is needed to improve the understanding of the contribution of hip PROM to pitching kinematics, joint loads, and injury risk. By identifying potential injurious risk factors than targeted interventions can be developed to reduce injury.

The current study's findings also showed hip IR, ER, and total PROM did not predict pelvis rotation during pitching. 
The results differ from a similar study in professional pitchers that reported decreased hip total PROM led to altered pelvis rotation during pitching. ${ }^{10}$ The discrepancy may indicate that PROM adaptations are more likely to appear in older pitchers. Older pitchers have accumulated more pitching exposure and time to elicit musculoskeletal adaptations. It is also possible that the timing of the season that the pitchers were assessed contributed to the different results between studies. The current study examined PROM in most pitchers prior to the beginning of fall practices and it is unclear when the professional pitchers were tested. If pitchers were tested in season, PROM patterns may be different due to the physical demands of participating in baseball. Stride leg and drive leg hip IR and stride leg total PROM have been observed to decrease over the course of a season in collegiate pitchers. ${ }^{28}$

Overall, total PROM in the current study (drive leg = $58.2^{\circ}$; stride leg $=57.0^{\circ}$ ) was approximately $46^{\circ}$ less than data presented in professional pitchers (drive leg $=94.8^{\circ}$; stride leg $=67 \cdot 0^{\circ}$ ). High school pitchers had approximately $37^{\circ}$ less drive leg total PROM than professional pitchers, whereas there was not a large discrepancy in stride leg motion. Differences in hip PROM measurement methodology may have contributed to the lack of agreement between studies. Robb et al. ${ }^{10}$ assessed hip PROM with the pitchers prone, whereas the current study assessed motion with the pitchers seated on a table. Assessing hip motion in a seated position can result in lower values due to compressive forces on the hip joint, so the results should be compared with caution. ${ }^{10}$ In addition, the current study used a digital inclinometer versus a bubble goniometer to assess PROM. Although different testing positions may result in different PROM magnitudes, the within-pitcher PROM asymmetry between studies is intriguing. Professional pitchers had asymmetry between legs which may explain why there were significant correlations with pitching kinematics that were not observed in the current study. Total PROM in the current study was similar to pilot data that has been presented in collegiate pitchers where the measurements were taken in a prone position. ${ }^{27}$ Drive leg total PROM in collegiate pitchers has been reported to range from $41.7^{\circ}-50.3^{\circ}$ while stride leg PROM ranges from $41.4^{\circ}-50.6^{\circ} .^{27,28}$ Additional PROM measures such as hip flexion, extension, abduction, and adduction could contribute to compensation in frontal and sagittal plane trunk mechanics and should be evaluated in future studies. It is possible that the trunk rotates less, compensations including increased trunk flexion or lateral flexion may occur in pitchers with limited hip PROM. Future studies should also aim to delineate the role of hip strength and lumbo-pelvic control as it relates to pitching mechanics and performance.

This study did have limitations that should be considered when interpreting the results. Data were collected on high school pitchers from a few high schools in a small geographical area in the southeastern United States, and the results may not be generalizable to pitchers from other geographical regions. The sample size was small, but it was similar to previous investigations into baseball biomechanics. $10,27,29,30$

\section{CONCLUSION}

Increased drive leg hip IR PROM was associated with decreased trunk rotation towards home plate. Hip total PROM and ER was not related to pitching mechanics. It is critical to continue identifying factors that contribute to altered mechanics to improve pitching performance and reduce injury rates. Range of motion is commonly measured by baseball organizations when performing player evaluations. If PROM was found to be related to altered pitching mechanics, training and rehabilitation programs could be developed to target specific deficits to improve performance and decrease injury susceptibility.

The Auburn University Institutional Review Board approved this study.

\section{CONFLICTS OF INTEREST}

The authors report no conflicts of interest.

\section{ACKNOWLEDGEMENTS}

This research was supported in part by an appointment to the postgraduate research program at the U.S. Army Aeromedical Research Laboratory administered by Oak Ridge Institute for Science and Education through an interagency agreement between the U.S. Department of Energy and the U.S. Army Medical Research and Development Command.

\section{DISCLAIMER}

The views, opinions, and/or findings contained in this report are those of the author(s) and should not be construed as an official Department of the Army position, policy, or decision, unless so designated by other official documentation. Citation of trade names in this report does not constitute an official Department of the Army endorsement or approval of the use of such commercial items.

Submitted: June 07, 2021 CDT, Accepted: August 17, 2021 CDT 


\section{REFERENCES}

1. Bunn JW. Scientific Principle of Coaching. Englewood Cliffs, NJ: Prentice-Hall Inc; 1972.

2. Putnam CA. A segment interaction analysis of proximal-to-distal sequential segment motion patterns. Med Sci Sports Exerc. 1991;23:130-144.

3. Sciascia A, Cromwell R. Kinetic chain rehabilitation: A theoretical framework. Rehabil Res Pract. 2012:1-9. doi:10.1155/2012/853037

4. Fortenbaugh D, Fleisig GS, Andrews JR. Baseball pitching biomechanics in relation to injury risk and performance. Sports Health. 2009;1(4):314-320.

5. Aguinaldo A, Escamilla R. Segmental power analysis of sequential body motion and elbow valgus loading during baseball pitching: Comparison between professional and high school baseball players. Orthop J Sports Med. 2019;7(2):2325967119827924. doi:10.1177/232596711 9827924

6. Laudner K, Wong R, Onuki T, Lynall R, Meister K. The relationship between clinically measured hip rotational motion and shoulder biomechanics during the pitching motion. J Sci Med Sport.

2015;18(5):581-584.

7. Anz AW, Bushnell BD, Griffin LP, Noonan TJ, Torry MR, Hawkins RJ. Correlation of torque and elbow injury in professional baseball pitchers. Am J Sports Med. 2010;38(7):1368-1374. doi:10.1177/0363546510 $\underline{363402}$

8. Aguinaldo AL, Buttermore J, Chambers H. Effects of upper trunk rotation on shoulder joint torque among baseball pitchers of various levels. J Appl Biomech. 2007;23:42-51.

9. Aguinaldo AL, Chambers H. Correlation of throwing mechanics with elbow valgus load in adult baseball pitchers. Am J Sports Med. 2009;37(10):2043-2048. doi:10.1177/03635465093367 $\underline{21}$

10. Robb AJ, Fleisig G, Wilk K, Macrina L, Bolt B, Pajaczkowski J. Passive ranges of motion of the hips and their relationship with pitching biomechanics and ball velocity in professional baseball pitchers. $\mathrm{Am}$ J Sports Med. 2010;38(12):2487-2493. doi:10.1177/036 3546510375535

11. Laudner KG, Moore SD, Sipes RC, Meister K. Functional hip characteristics of baseball pitchers and position players. Am J Sports Med. 2010;38(2):383-387. doi:10.1177/0363546509347365
12. Oliver GD, Weimar WH, Henning L. Effects of a simulated game on muscle activaiton in youth baseball pitchers. J Strength Cond Res. 2016;30(2):415-420.

13. Harding JL, Picha KJ, Huxel Bliven KC. Pitch volume and glenohumeral and hip motion and strength in youth baseball pitchers. J Athl Train. 2018;53(1):60-65.

14. Saito M, Kenmoku T, Kameyama K, et al. Relationship between tightness of the hip joint and elbow pain in adolescent baseball players. Orthop J Sports Med. 2014;2(5):232596711453242.

15. Sekiguchi T, Hagiwara Y, Yabe Y, et al. Restriction in hip internal rotation of the stride leg is assocaite with elbow and shoulder pain in elite young baseball players. J Shoulder Elbow Surg. 2020;29:139-145.

16. Ellenbecker TS, Ellenbecker GA, Roetert EP, Silva RT, Keuter G, Sperling F. Descriptive profile of hip rotation range of motion in elite tennis players and professional baseball pitchers. Am J Sports Med. 2007;35(8):1371-1376. doi:10.1177/036354650730026 $\underline{0}$

17. Sauers EL, Huxel Bliven KC, Johnson MP, Falsone $\mathrm{S}$, Walters S. Hip and glenohumeral rotational range of motion in healthy professional baseball pitchers and position players. Am J Sports Med. 2013;42(2):430-436. doi:10.1177/0363546513508537

18. Scher S, Anderson K, Weber N, Bajorek J, Rand K, Bey MJ. Associations among hip and shoulder range of motion and shoulder injury in professional baseball players. J Athl Train. 2010;45(2):191-197. do i:10.4085/1062-6050-45.2.191

19. Wu G, Siegler S, Allard P, et al. ISB recommendation on definitions of joint coordinate systems of various joints for the reporting of human joint motion-Part I: Ankle, hip, and spine. J Biomech. 2002;35:543-548.

20. Wu G, van der Helm FCT, Veeger HEJ, et al. ISB recommendation on definitions of joint coordinate systems of various joints for the reporting of human joint motion-Part II: shoulder, elbow, wrist and hand. J Biomech. 2005;38(5):981-992. doi:10.1016/i.jbi omech.2004.05.042

21. Bell AL, Pedersen DR, Brand RA. A comparison of the accuracy of several hip center location prediction methods. J Biomech. 1990;23(6):617-621. 
22. Biryukova EV, Roby-Brami A, Frolov AA, Mokhtari M. Kinematics of human arm reconstructed from spatial tracking system recordings. J Biomech. 2000;33(8):985-995.

23. Camomilla V, Cereatti A, Vannozzi G, Cappozzo A. An optimized protocol for hip joint centre determination using the functional method. $J$ Biomech. 2006;39(6):1096-1106. doi:10.1016/j.jbiome ch.2005.02.008

24. Barfield J, Anz AW, Andrews J, Oliver GD. Relationship of glove arm kinematics with established pitching kinematic and kinetic variables among youth baseball pitchers. Orthop J Sports Med. 2018;6(7):1-6. doi:10.1177/2325967118784937

25. Wasserberger KW, Barfield JW, Downs JL, Oliver GD. Glenohumeral external rotation weakness partially accounts for increased humeral rotation torque in youth baseball pitchers. J Sci Med Sport. 2020;23(4):361-365.

26. Oliver GD, Weimar W. Hip range of motion and scapula position in youth baseball pitching pre and post simulated game. J Sports Sci.

2015;33(14):1447-1453. doi:10.1080/02640414.2014.9 $\underline{90493}$
27. Zeppieri Jr G, Bruner ML, Michelini JP, Farmer KW. The relationship between hip range of motion and pitching kinematics related to increased elbow valgus loads in collegiate baseball pitchers: A pilot study. Int J Sports Phys Ther. 2021;16(2):468-476. doi:10.26603/ 001c.21319

28. Zeppieri G Jr, Lentz TA, Moser MW, Farmer KW. Changes in hip range of motion and strength in collegiate baseball pitchers over the course of a competitive season: A pilot study. Int J Sports Phys Ther. 2015;10(4):505-513.

29. Fleisig GS, Bolt B, Fortenbaugh D, Wilk KE, Andrews JR. Biomechanical comparison of baseball pitching and long-toss: implications for training and rehabilitation. J Orthop Sports Phys Ther. 2011;41(5):296-303. doi:10.2519/jospt.2011.3568

30. Fleisig GS, Diffendaffer AZ, Ivey B, et al. Changes in youth baseball pitching biomechanics: A 7-Year longitudinal study. Am J Sports Med. 2017;46(1):44-51. doi:10.1177/0363546517732034 